\title{
An Unusual Metastatic Pattern of a Transverse Colon Cancer: A Case Report and Review of Literature
}

\author{
Tallat Ejaz ${ }^{1 *}$, Shahbaz Mansoor ${ }^{1,2}$ and Eltaib Saad ${ }^{1}$ \\ ${ }^{1}$ Department of Surgery, Midland Regional Hospital Mullingar, Mullingar, Co-Westmeath, Republic of Ireland \\ ${ }^{2}$ Honorary Clinical Associate Professor, Maastricht University and Royal College of Surgeons in Ireland (RCSI) - Dublin
}

Received: January 01, 2019; Published: January 31, 2019

*Corresponding author: Tallat Ejaz FRCSI, General and Pediatric Surgeon, Midland Regional Hospital Mullingar

\begin{abstract}
Colorectal cancer is the second most common cancer in females and the third in males worldwide. The most common sites of colorectal cancer metastasis are the liver, lungs, peritoneum, brain, and bones. Metastasis to para-aortic, mediastinal and left supraclavicular lymph nodes without visceral involvement is fairly rare. We present a rare case of a transverse colon cancer in a young patient with an unusual metastatic pattern to para-aortic, anterior mediastinal and left supraclavicular nodes without hepatic, pulmonary or bony metastasis. The patient's main complaint was a left supraclavicular swelling for 3 months. Notably, he had no specific symptoms to suggest colonic tumor. Left supraclavicular node biopsy revealed metastatic glandular tumor with a likely primary colorectal origin. A colonoscopy showed a mass at the proximal transverse colon and CT scan of the chest, abdomen and pelvis revealed a locally advanced transverse colon tumor with involved regional nodes and a distant metastasis in para-aortic, anterior mediastinal and left supraclavicular lymph nodes. No liver, lungs or bone metastasis noted. He underwent an extended right hemicolectomy and was referred to oncology team for palliative chemotherapy.
\end{abstract}

Keywords: Colonic cancer; Left supraclavicular lymph node; Solid organs metastasis; Distant metastasis; Unusual pattern

\section{Introduction}

\section{Learning Objectives}

a. Left supraclavicular lymphadenopathy can be the first manifestation of an advanced colonic cancer, even in absence of typical clinical features of colonic cancers.

b. Systemic metastasis of a colonic tumor to paraaortic, anterior mediastinal and left supraclavicular lymph nodes without solid visceral (the liver, lungs and bones) involvement is a rare oncological entity but may occur as demonstrated in this case.

\section{Background}

Colorectal cancer (CRC) is the second most common cancer in females and the third in males worldwide [1]. The literature reported a steady increase in the incidence of CRC among both males and females under the age of 50 at a rate of $2.1 \%$ per year from 1992 through 2012 [2]. Nevertheless, screening is not currently recommended for individuals under the age of 50 unless they have inflammatory bowel disease, a positive family history, or a predisposing inherited syndrome [3]. CRCs spread principally through lymphatic and hematogenous routes, and less commonly by contiguous and trans-peritoneal dissemination [2]. Approximately $20 \%$ of patients in the United States have a distant metastatic disease at the time of presentation [2]. The most common reported metastatic sites are the liver, lungs, brain, peritoneum, and bones [2]. Most CRCs (60\%) eventually develop metastatic disease, with liver involvement alone accounts for almost $50 \%$ of systemic metastasis [4].

Metastasis to mediastinal and left supraclavicular lymph nodes without solid organs involvement is a fairly rare oncological entity [5-9]. We present a rare case of a transverse colon cancer in a young patient with an unusual pattern of metastasis to paraaortic, anterior mediastinal and left supraclavicular lymph nodes without solid organs (liver, lungs and bones) metastasis. The patient's chief complaints were a left supraclavicular swelling and weight loss for 3 months. Interestingly, he didn't report any abdominal pain, recent bowel habits changes, rectal bleeding or anemia symptoms. A few reports in the reviewed literature described the similar pattern observed in this presented case [6-9].

\section{Case Presentation}

A 45-year-old Caucasian male was referred to our clinic with a 3-month history of left supraclavicular swelling and unintentional weight loss. His past medical history was significant for gastritis 7 years ago which was responsive to proton pump inhibitors (PPI) courses. He had no voice changes, epistaxis, difficulty or pain during swallowing, epigastric pain, 
nausea or vomiting. He didn't have abdominal pain, recent bowel habits changes, or rectal bleeding. Additionally, he had no cough, chest pain, haemoptysis or shortness of breath. The patient also denied fever, chills, night sweating, rash, pruritus or bruising. The rest of the systemic review was otherwise unremarkable. His family history was significant for gastric cancer. He was a social drinker, but he never smoked.

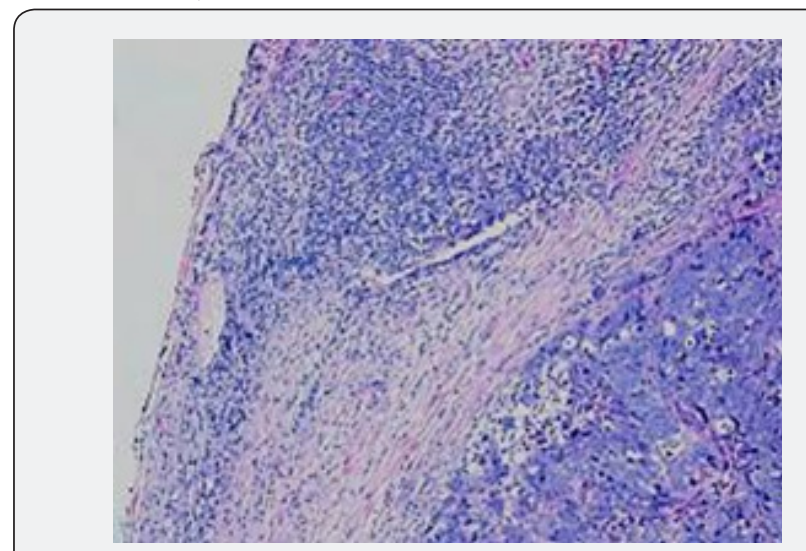

Figure 1: Hematoxylin and Eosin stained slide of the left supraclavicular lymph node showing metastatic poorlydifferentiated glandular tumor cells (high power X200).

Physical examination was significant for an enlarged (4X4) $\mathrm{cm}$ firm and fixed left supraclavicular lymph node (Troisier's sign). Cervical, axillary and inguinal lymph nodes were not clinically palpable. Abdominal examination revealed a soft and non-tender abdomen with no palpable masses. There was no hepatomegaly or splenomegaly. The rest of the systemic was essentially normal. General blood tests revealed a low $\mathrm{Hb}$ $(10.5 \mathrm{~g} / \mathrm{dl})$ with normal total and differential WCC and platelets counts. Renal and liver profiles were within the normal ranges. His CXR was normal. He underwent a left supraclavicular lymph node biopsy under anesthesia. Histological examination of node tissues showed a metastatic poorly-differentiated malignant tumor with glandular components (Figure 1).

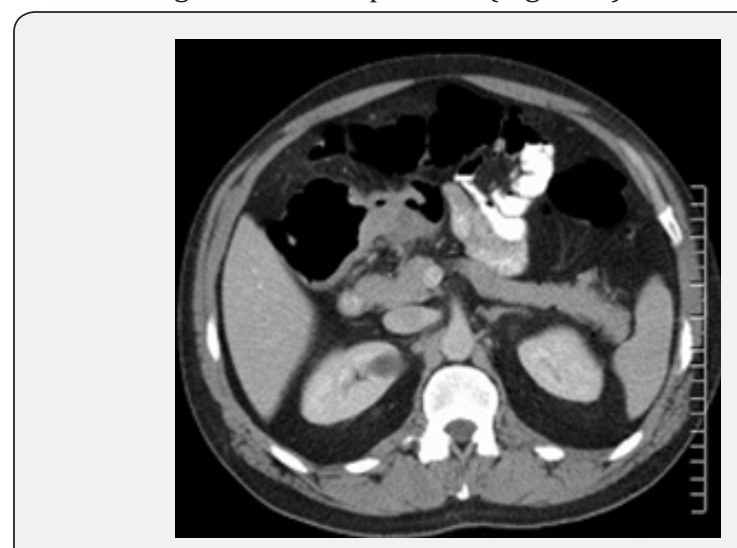

Figure 2: Axial CT scan of the chest, abdomen and pelvis with IV and oral contrast showing a locally invasive tumor at the proximal transverse colon.

Immunohistochemistry revealed a strong positivity for AE1/ AE3 and Cytokeratin 20 (CK20), and PASD stained for mucin, but the staining for Cytokeratin 7 (CK7), p63, and TTF-1 was negative. The findings suggested a likely primary colorectal origin. He underwent an urgent OGD and full colonoscopy. OGD revealed a normal esophageal mucosa and mild gastritis which tested negative for $\mathrm{H}$. pylori. Full colonoscopy detected a partially-obstructing circumferential mass at the proximal transverse colon near the hepatic flexure. The biopsy report from this colonic mass confirmed an invasive poorly-differentiated colonic adenocarcinoma and the findings were almost identical to the left supraclavicular node's biopsy. The patient had a computed tomography (CT) scan of the chest, abdomen and pelvis which depicted a locally advanced proximal transverse colon tumor spreading well beyond the serosa posteriorly with local infiltration of pericolic fat and regional lymphadenopathy Figure 2-A. There was a significant metastasis in the para-aortic nodes Figure 2-B, anterior mediastinal nodes Figure 2-C and left supraclavicular nodes Figure 2-D. No evidence of hepatic, pulmonary or bony metastasis noted. Carcinoembryonic antigen (CEA) level was above the normal $(22 \mathrm{ng} / \mathrm{ml}$, normal reference $<3.5 \mathrm{ng} / \mathrm{ml}$ ). Alpha-fetoprotein (AFP) level was within the normal ranges.
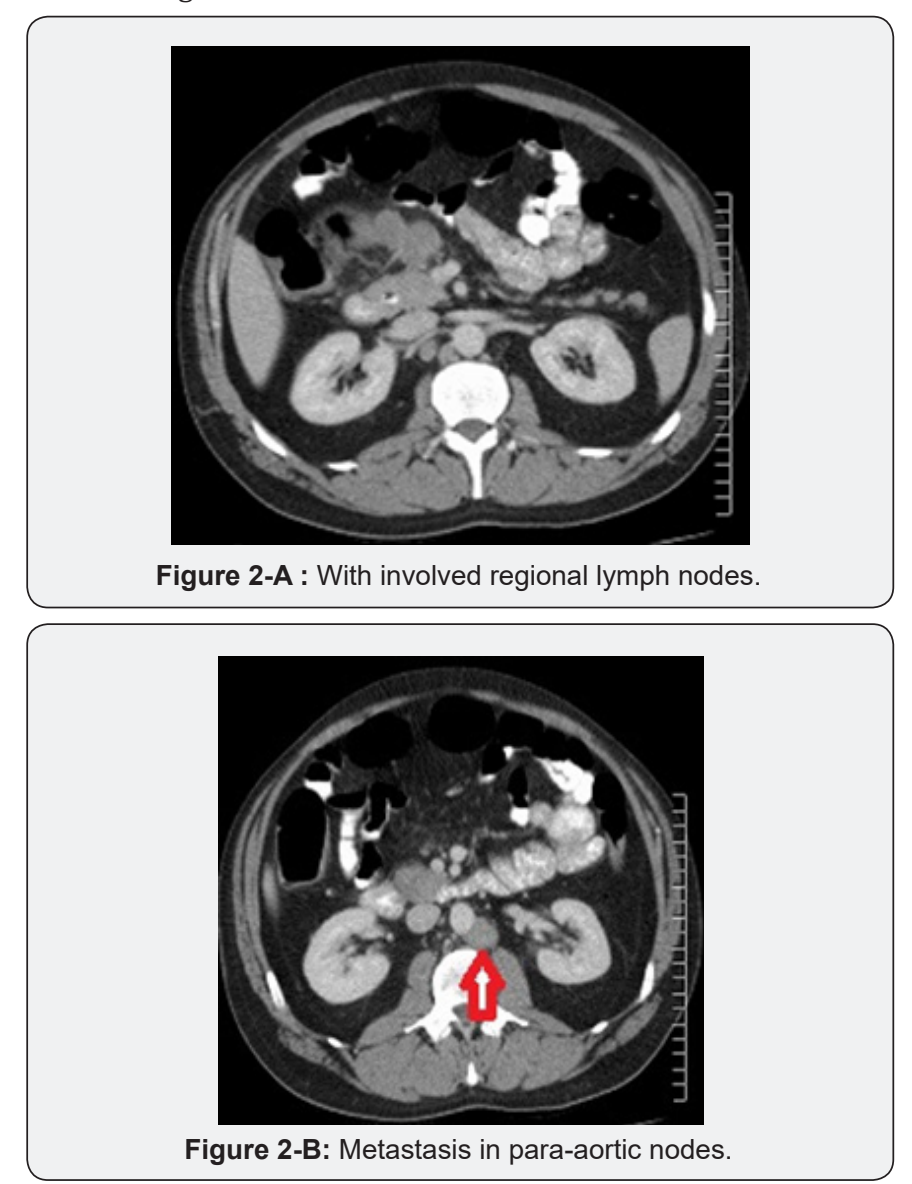

A multidisciplinary team (MDT) meeting advised for a tumor resection as the patient was at a high risk of a large bowel obstruction, and a palliative chemotherapy for the advanced metastatic disease. He underwent a laparoscopic-assisted extended right hemicolectomy with an uneventful perioperative course. Histopathology of the surgical specimen revealed a poorly-differentiated transverse colon adenocarcinoma Figure 3A-3B with a full-thickness invasion of the bowel wall and 
extensive infiltration of pericolic fat. 2 out of 20 lymph nodes examined were positive with extensive lympho-vascular invasion and positive mesenteric margins. The staging was stage IV (T4N1M1). KRAS assay returned wild. He was referred to the oncology team for palliative chemotherapy. The patient was treated with cycles of XELOX regimen (capecitabine plus oxaliplatin) with Erbitux (cetuximab, C225). Initial follow-up CT scans of the neck, chest and abdomen after 4 months revealed a positive response to chemotherapy with acceptable diminution of the mediastinal and para-aortic lymph nodes, but a stable left supraclavicular node status. The patient was alive for 2 years following the extended right hemicolectomy.
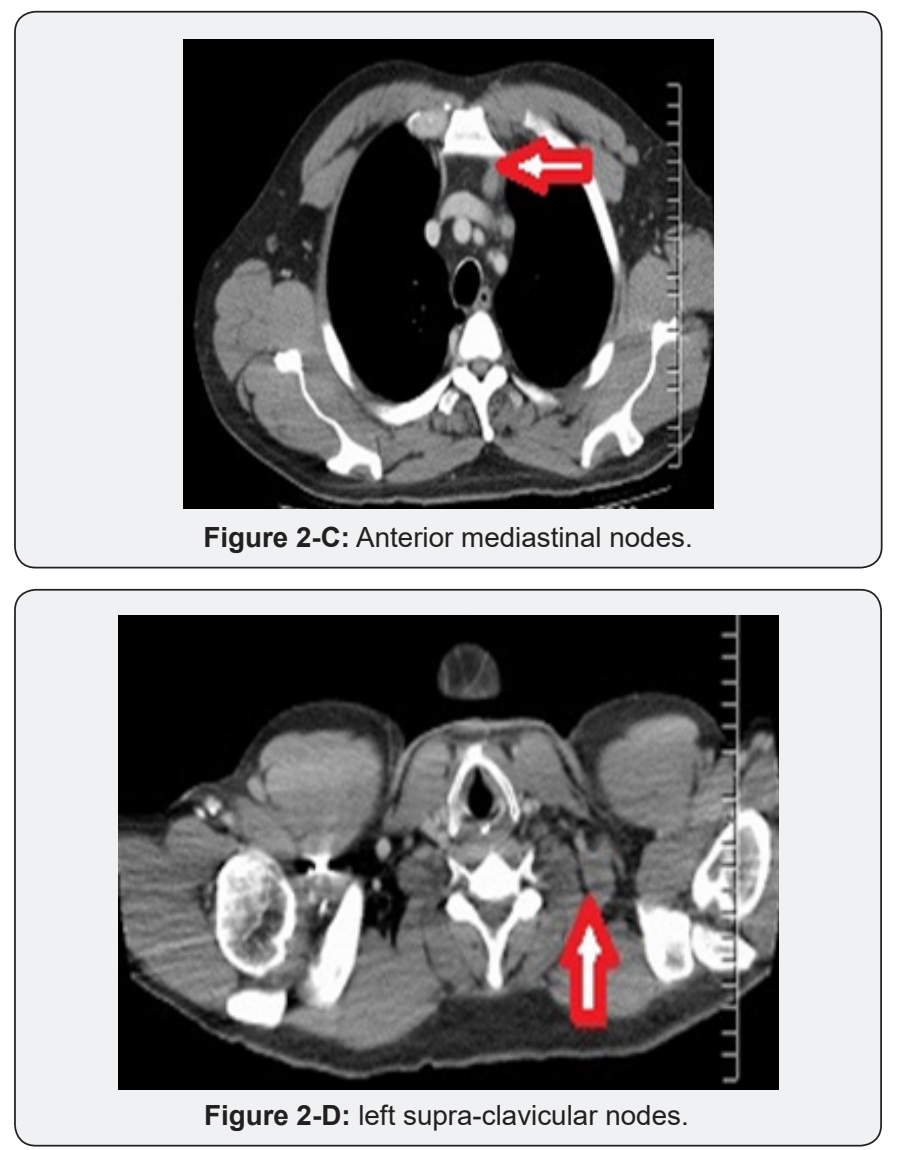

Discussion

Despite increasing uptake of CRC through screening programs to detect asymptomatic stage, most CRCs (70 to 90 $\%$ in recent series) are diagnosed after the onset of symptoms [10]. The symptoms of colonic tumors would vary according to the location and the size of the primary tumor. Right-sided carcinomas are usually polypoid or ulcerative lesions that present with abdominal pain (60\%), chronic blood loss (48.5\%), anemia $(21.3 \%)$, abdominal mass $(24 . \%)$ or obstruction if they occlude the lumen (17\%), while left-sided carcinomas are commonly obstructive rings that present with bowel habits changes (48\%), diarrhea (12\%) or large bowel obstruction [4].

In addition, non-specific symptoms, for instance, weight loss and anorexia occur in less than one-third of patients $(28.8 \%)$. Additionally, about $2.5 \%$ of patients are asymptomatic at the time of diagnosis [4]. Interestingly enough, our patient had no specific symptoms to suggest a colonic tumor, although the tumor had resulted in a remarkable narrowing of the lumen near the hepatic flexure as noted on CT scan findings, and he was at a high risk of a large bowel obstruction. Almost $20 \%$ of CRC patients in the United States would have a distant metastatic disease at the initial time of presentation [2]. The liver, lungs, peritoneum, brain, and bones are the most frequently reported extra-abdominal metastatic sites [2,4], with the liver involvement alone accounts for almost $50 \%$ of systemic metastasis [4] and nearly $85 \%$ in other series of 89 patients with metastatic (stage IV) disease [11]. The metastatic pattern observed in this case without liver or lungs involvement thus represents an oncological rarity.

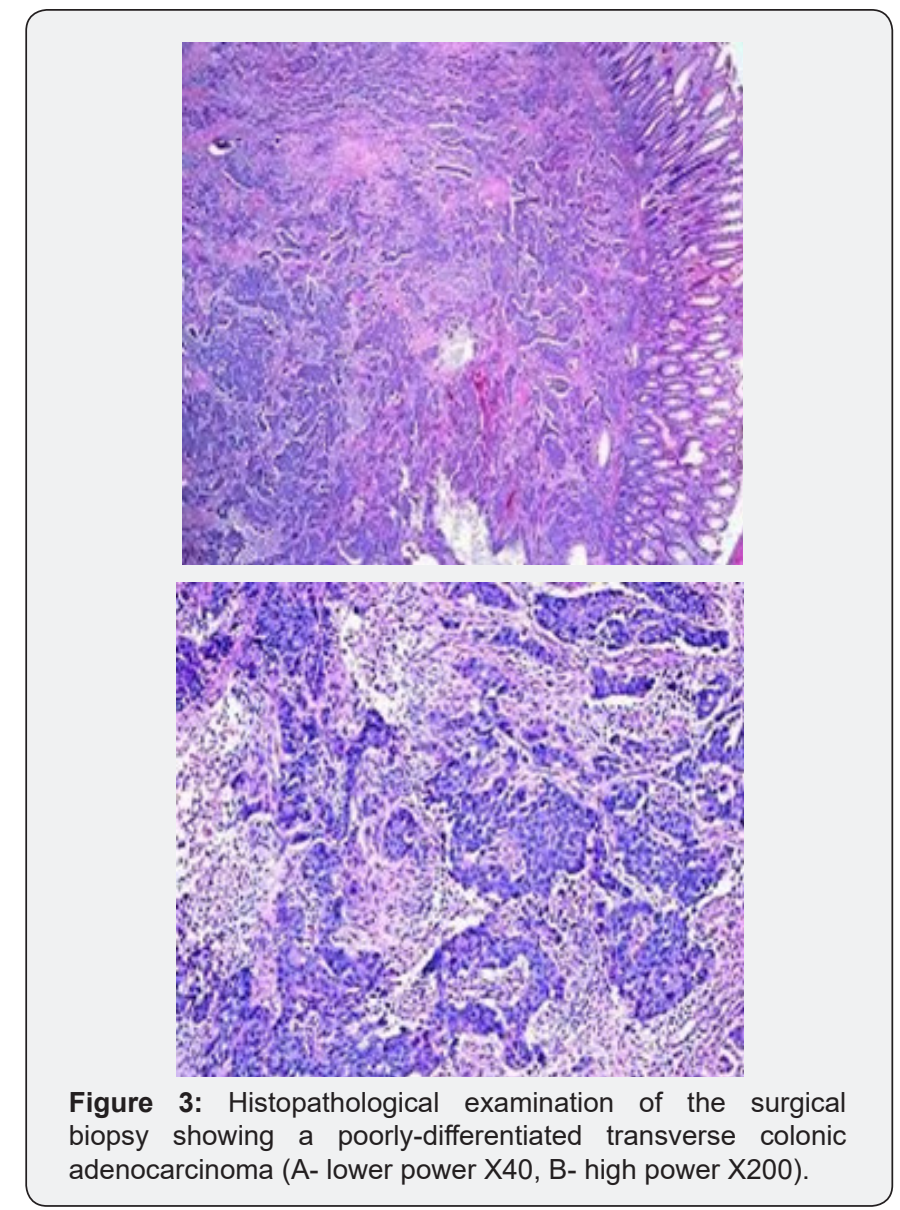

Distal metastasis of colorectal tumors to mediastinal lymph nodes without lung metastasis is an exceedingly rare entity $[5,12$ 15]. In fact, Kousa et al. mentioned only 7 cases of metachronous mediastinal lymph node metastasis from colorectal cancers in the English literature, including his reported one [15]. Nevertheless, the exact route of mediastinal lymph node metastasis with colon cancers is not yet known $[5,13,15]$. It has been postulated that involvement of the mediastinal lymph nodes is through the para-aortic lymphatic drainage route towards the thoracic duct [13]. This presumed theory might explain the metastasis to the posterior mediastinal nodes group due to their close proximity to (and their eventual drainage) to the thoracic duct, but would not justify the involvement of the anterior mediastinal lymph 
nodes group (as occurred in our patient), which are however, well-distant from the thoracic duct and they don't usually drain to it as there is no direct communication between the thoracic duct and Broncho mediastinal trunk that drain these lymph nodes group [15].

Nevertheless, MacLoud et al. [16] suggested a retrograde reflux of tumor micro-emboli from the thoracic duct into the Broncho mediastinal trunk through incompetent lymphatics valves and this would possibly explain the anterior mediastinal lymph nodes involvement. Cervical and left supraclavicular lymphadenopathy is well-known to be the first manifestation of colorectal tumors [6,7]. Nevertheless, the occurrence of distant cervical and supraclavicular nodes metastasis without solid organs metastasis is typically rare and was reported only in a few cases in the reviewed literature [6-9]. It has been suggested that after the metastatic involvement of para-aortic lymph nodes, a sequential left supraclavicular metastasis occurs as a result of infiltration of the thoracic duct with tumor cells with formation of skip metastasis between the regional lymph node stations and the distal nodes which are considered as end-nodes of the lymphatic pathway [6].

\section{Conclusion}

We report an unusual metastatic pattern of a transverse colonic tumor to the anterior mediastinal and supraclavicular lymph nodes without liver or lungs involvement. In this reported case, the patient's main complaint was a left supraclavicular swelling and he had no colonic cancer-suggesting symptoms, though the tumor had caused a remarkable luminal narrowing with extensive local invasion as noted on CT scan findings. This case also demonstrates the clinical significance of enlarged left supraclavicular node (Virchow's node) which denotes a distant metastasis from aero-digestive malignancies; a critical finding that should warrant an urgent work-up to search for the primary cancer.

\section{References}

1. Jemal A, Bray F, Center MM, Ferlay J, Ward E, et al. (2011) Global cancer statistics. CA Cancer J Clin 61(2): 69-90.

2. Siegel RL, Miller KD, Jemal A (2016) Cancer statistics. CA Cancer J Clin 66: 7.

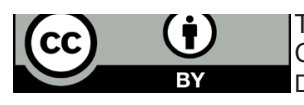

This work is licensed under Creative Commons Attribution 4.0 Licens DOI: 10.19080/OAJS.2019.10.555785
3. Dozois EJ, Boardman LA, Suwanthanma W, Limburg PJ, Cima RR, et al. (2008) Young-onset colorectal cancer in patients with no known genetic predisposition: can we increase early recognition and improve outcome? Medicine (Baltimore) 87(5): 259-263.

4. Achmad H, Hanifa R (2015) Supraclavicular Lymph nodes: Unusual Manifestation of Metastases Adenocarcinoma Colon. Acta Med Indones 47(4): 333-339.

5. Iwata T, Chung K, Hanada S, Toda M, Nakata K, et al. (2013) Solitary bulky mediastinal lymph node metastasis from colon cancer. Ann Thorac Cardiovasc Surg 19(4): 313-315.

6. Aksel B, Dogan L, Karaman N, Demirci S (2013) Cervical lymphadenopathy as the first presentation of sigmoid colon cancer. Middle East J Cancer 4(4): 185-188.

7. Basso L, Izzo L, Calisi E, Cavallaro G, Costi U, et al. (2007) Cervical node metastasis as the first sign of cancer of the caecum. Anticancer Res 27(5B): 3589-3592.

8. Hirose H, Ikeda M, Miyoshi N, Kim HM, Okano M, et al. (2010) A longterm survival case of rectal cancer with Virchow's lymph node metastasis by multimodality therapy. Gan To Kagaku Ryoho 37(12): 25452547.

9. Dillip Kumar Parida, Sovan Sarang Dhar (2018) Supraclavicular Lymph Node Metastasis from Colon Cancer without Involvement of Liver and Lungs. Acta Scientific Cancer Biology 2(5): 14-16.

10. Moiel D, Thompson J (2011) Early detection of colon cancer-the Kaiser Permanente Northwest 30-year history: how do we measure success? Is it the test, the number of tests, the stage, or the percentage of screen-detected patients? Perm J 15(4): 30-38.

11. Scoggins CR, Meszoely IM, Blanke CD, Beauchamp RD, Leach SD (1999) Non-operative management of primary colorectal cancer in patients with stage IV disease. Ann Surg Oncol 6(7): 651-657.

12. Hess KR, Varadhachary GR, Taylor SH, Wei W, Raber MN, et al. (2006) Metastatic patterns in adenocarcinoma. Cancer 106(7): 1624-1633.

13. El Halabi MM, Chaaban SA, Meouchy J, Page S, Salyers WJ (2014) Colon cancer metastasis to mediastinal lymph nodes without liver or lung involvement: A case report. Oncol Lett 8(5): 2221-2224.

14. Musallam KM, Taher AT, Tawil AN, Chakhachiro ZI, Moh'd Z H, et al. (2008) Solitary mediastinal lymph node metastasis in recto-sigmoid carcinoma: a case report. Cases J 1(1): 69.

15. Toda K, Kawada K, Sakai Y, Izumi H (2017) Metachronous mediastinal lymph node metastasis from ascending colon cancer: A case report and literature review. Int J Surg Case Rep 41: 336-339.

16. McLoud TC, Kalisher L, Stark P, Greene R (1978) Intrathoracic lymph node metastases from extrathoracic neoplasms. AJR Am J Roentgenol 131(3): 403-407.

\section{Your next submission with Juniper Publishers will reach you the below assets}

- Quality Editorial service

- Swift Peer Review

- Reprints availability

- E-prints Service

- Manuscript Podcast for convenient understanding

- Global attainment for your research

- Manuscript accessibility in different formats

( Pdf, E-pub, Full Text, Audio)

- Unceasing customer service

Track the below URL for one-step submission https://juniperpublishers.com/online-submission.php 